\title{
The weighted discrete dynamic inequalities for 4-convex functions, and its generalization on time scales with constant graininess function
}

\author{
Hira Ashraf Baig ${ }^{1 *}$ (D) and Naveed Ahmad ${ }^{1}$
}

\section{"Correspondence:}

habaig@iba.edu.pk

'Department of Mathematical

Sciences, Institute of Business

Administration, Karachi, Pakistan

\begin{abstract}
Motivated by Pečarić et al. in (J. Math. Inequal. 11(2):543-5502017), we established here weighted discrete dynamic inequalities for the difference of second divided difference of 4-convex functions. Further we extend and unify the two inequalities, by establishing the theory of $n$-convexity on time scales having constant graininess function.
\end{abstract}

MSC: 26D20; 39B62; 36A51; 34N05

Keywords: The weighted delta-integral estimates; 4-convex function; Time scales

\section{Introduction}

The two well-known equations of difference and differential equations were born due to the sets of integers and real numbers, respectively. By virtue of a comprehensive study of these kinds of equations, two different mathematical disciplines came into being, known as discrete and continuous analysis. Many productive attempts have been made to bring the two concepts together. Among those, the most significant attempt towards the unification is considered to be the time scale theory. This theory was developed by Aulbach and Hilger [2]. The pioneer of the theory provided efficient tools to unify discrete and continuous problems in his newly introduced theory. The classicist theories on an arbitrary non-empty closed subset $\mathbb{T}$ of real numbers were extended to a set designated as time scale. The two well-known particular cases of time scales are the set of integers $\mathbb{Z}$ and the set of real numbers $\mathbb{R}$. The applications of this theory have captured the attention of many prolific researchers over the past few years. It has different applications in engineering, biology, social sciences, neutral network, physics, and economics. For additional details and basic notions of time scale calculus, we refer to [3-5].

Study and applications of convex functions and their different classes have a significant place in the literature. Here we will discuss a special class of convex functions, known as $n$-convex functions. The $n$-convexity or higher order convexity, for $n \geq 3$, on an interval was first considered by Hopf [6] in his dissertation. This was further studied in great detail

(c) The Author(s) 2020. This article is licensed under a Creative Commons Attribution 4.0 International License, which permits use sharing, adaptation, distribution and reproduction in any medium or format, as long as you give appropriate credit to the original author(s) and the source, provide a link to the Creative Commons licence, and indicate if changes were made. The images or other third party material in this article are included in the article's Creative Commons licence, unless indicated otherwise in a credit line to the material. If material is not included in the article's Creative Commons licence and your intended use is not permitted by statutory regulation or exceeds the permitted use, you will need to obtain permission directly from the copyright holder. To view a copy of this licence, visit http://creativecommons.org/licenses/by/4.0/. 
by Popoviciu in $[7,8]$. A detailed review of this class of functions is given in $[9,10]$. Convex functions have been defined on time scales by Dinu in 2008 [11]; after that large numbers of inequalities for convex functions on time scales have been developed; see for example $[12,13]$. To the best of our knowledge $n$-convexity has not been defined on time scales yet. In [14] Mikić and Pečarić developed some integral inequalities on time scales that hold for $n$-convex functions but they defined their function on an interval on $\mathbb{R}$. We need to develop $n$-convex functions on time scales, in order to proceed towards our major results.

This paper is organized as follows. In Sect. 2 we give some preliminaries supporting to the central results. Section 3.1 is devoted to defining $n$-convexity on time scales and establishing a relationship between the $n$th divided difference and the $n$th ordered delta derivatives on time scales. We develop discrete dynamic inequalities for the difference of the second divided difference of 4-convex functions in Sect. 3.2. Finally in Sect. 3.3 we unify the inequalities defined in the preceding section, along with their continuous versions established in [1] through the theory of time scales calculus.

\section{Preliminaries}

We accumulate in this section some fundamental preliminaries that are used throughout the remaining part of the paper.

\subsection{Time scale calculus}

This part consists of the following basic concepts of time scales related to this article.

We quote [3]: "A time scale is defined to be an arbitrary closed subset of the real numbers $\mathbb{R}$, with the standard inherited topology. Since a time scale may or may not be connected, we need the concept of jump operators, the forward jump operator and the backward jump operator are defined by $\sigma(\breve{l}):=\inf \{s \in \mathbb{T}: s>\breve{l}\}$, and $\rho(\breve{l}):=\sup \{s \in \mathbb{T}: s<\breve{l}\}$, where $\inf \phi=\sup \mathbb{T}$ and $\sup \phi=\inf \mathbb{T}$. Thus, we classify the points $\breve{\iota} \in \mathbb{T}$ in such a manner that, if $\sigma(\breve{l})>\breve{\iota}$, then $\breve{\iota}$ is right-scattered, and if $\rho(\breve{l})<\breve{\iota}$, then $\breve{\iota}$ is left-scattered. Points that are right-scattered and left-scattered at the same time are called isolated. Also, if $\sigma(\breve{l})=\breve{\iota}$, then $\breve{\iota}$ is said to be right-dense, and if $\rho(\breve{\iota})=\breve{\iota}$, then $\breve{\iota}$ is said to be left-dense. Points that are simultaneously right-dense and left-dense are called dense. The mappings $\mu, v: \mathbb{T} \longrightarrow$ $[0, \infty)$ defined by

$$
\mu(\breve{l})=\sigma(\breve{l})-\breve{\iota} \quad \text { and } \quad v(\breve{l})=\Delta \breve{\iota}-\rho(\breve{l})
$$

are called the forward and backward graininess functions respectively."

Throughout this paper, an interval in time scales is denoted by $I_{\mathbb{T}}=I \cap \mathbb{T}$, where $I \subseteq \mathbb{R}$. We define

$$
\mathbb{T}^{\mathcal{K}}= \begin{cases}\mathbb{T} \backslash S^{+} & \text {if } \mathbb{T} \text { acquire a left-scattered maximum } S^{+}, \\ \mathbb{T} & \text { otherwise. }\end{cases}
$$

Definition 2.1 ([3]) Assume $u: \mathbb{T} \rightarrow \mathbb{R}$ is a function and let $\breve{\iota} \in \mathbb{T}^{\mathcal{K}}$. Then we define $u^{\Delta}(\breve{l})$ to be the number (provided it exists) with the property that, given any $\epsilon>0$, there is a neighborhood $U_{\mathbb{T}}$ of $\breve{\iota}$ such that

$$
\left|(u(\sigma(\breve{l}))-u(s))-u^{\Delta}(\breve{l})[\sigma(\breve{\iota})-s]\right| \leq \epsilon|\sigma(\breve{l})-s| \quad \text { for all } s \in U_{\mathbb{T}}
$$


We call $u^{\Delta}(\breve{\iota})$ the delta derivative of $u$ at $\breve{l}$. We say that $u$ is delta differentiable on $\mathbb{T}^{\mathcal{K}}$ provided $u^{\Delta}(\breve{\iota})$ exists for all $\breve{\iota} \in \mathbb{T}^{\mathcal{K}}$.

Theorem 2.2 ([3]) For all $\breve{\iota} \in \mathbb{T}^{\mathcal{K}}$, we have the following:

a. If $u$ is delta differentiable at $\breve{\iota}$, then $u$ is continuous at $\breve{\imath}$.

b. If $u$ is continuous at $\breve{\iota}$ and $\breve{\iota}$ is right-scattered, then $u$ is delta differentiable at $\breve{\iota}$ with

$$
u^{\Delta}(\breve{l})=\frac{u(\sigma(\breve{l}))-u(\breve{l})}{\mu(\breve{l})}
$$

c. If $\breve{\iota}$ is right-dense, then $u$ is delta differentiable at $\breve{\iota}$ if and only if $\lim _{s \rightarrow \breve{\iota}} \frac{u(\breve{\imath})-u(s)}{\breve{\imath}-s}$ exists as a finite number. In this case,

$$
u^{\Delta}(\breve{l})=\lim _{s \rightarrow \breve{\iota}} \frac{u(\breve{\iota})-u(s)}{\breve{\iota}-s} .
$$

d. If $u$ is delta differentiable at $\breve{\iota}$, then $u(\sigma(\breve{l}))=u(\breve{l})+\mu(\breve{l}) u^{\Delta}(\breve{l})$.

Theorem 2.3 ([3]) Assume $u, v: \mathbb{T} \rightarrow \mathbb{R}$ are $\Delta$-differentiable at $\breve{\iota} \in \mathbb{T}^{\mathcal{K}}$, then:

a. The sum, $u+v$ is $\Delta$-differentiable at $\breve{\iota}$, and

$$
(u+v)^{\Delta}(\breve{l})=u^{\Delta}(\breve{l})+v^{\Delta}(\breve{l}) .
$$

b. For any constant $k$, $k u$ is $\Delta$-differentiable at $\breve{\iota}$, with

$$
(k u)^{\Delta}(\breve{l})=k u^{\Delta}(\breve{l})
$$

c. The product $u v$ is $\Delta$-differentiable at $\breve{\iota}$ with

$$
(u v)^{\Delta}(\breve{l})=u^{\Delta}(\breve{l}) v(\breve{l})+u(\sigma(\breve{l})) v^{\Delta}(\breve{l})=v^{\Delta}(\breve{l}) u(\breve{l})+v(\sigma(\breve{l})) u^{\Delta}(\breve{l}) .
$$

d. If $u(\breve{l}) u(\sigma(\breve{l})) \neq 0$, then $\frac{1}{u}$ is $\Delta$-differentiable at $\breve{\iota}$ with

$$
\left(\frac{1}{u}\right)^{\Delta}(\breve{\imath})=-\frac{u^{\Delta}}{u(\breve{l}) u(\sigma(\breve{\imath}))}
$$

Definition 2.4 ([3]) A function $U: \mathbb{T} \rightarrow \mathbb{R}$ is called a delta anti derivative of $u: \mathbb{T} \rightarrow \mathbb{R}$ if $U^{\Delta}(\breve{l})=u(\breve{l})$ for all $\breve{\iota} \in \mathbb{T}^{\mathcal{K}}$. Then we define the delta integral by

$$
\int_{t_{a}}^{\breve{\iota}} u(s) \Delta s=U(\breve{l})-U\left(t_{a}\right) .
$$

Definition 2.5 ([3]) A function $u: \mathbb{T} \rightarrow \mathbb{R}$ is called rd-continuous provided it is continuous at right-dense points in $\mathbb{T}$ and its left-sided limits exist (finite) at left-dense points in $\mathbb{T}$. The set of rd-continuous functions $u: \mathbb{T} \rightarrow \mathbb{R}$ is denoted by

$$
C_{\mathrm{rd}}=C_{\mathrm{rd}}(\mathbb{T})=C_{\mathrm{rd}}(\mathbb{T}, \mathbb{R})
$$


The set consisting of functions $u: \mathbb{T} \longrightarrow \mathbb{R}$ which are differentiable and whose derivative is rd-continuous is denoted by

$$
C_{\mathrm{rd}}^{1}=C_{\mathrm{rd}}^{1}(\mathbb{T})=C_{\mathrm{rd}}^{1}(\mathbb{T}, \mathbb{R}) .
$$

Theorem 2.6 ([3]) If $t_{a}, t_{b}, t_{c} \in \mathbb{T}, \alpha \in \mathbb{R}$ and $u, v \in C_{\mathrm{rd}}$, then
a. $\int_{t_{a}}^{t_{b}}[u(\breve{l})+v(\breve{l})] \Delta \breve{\iota}=\int_{t_{a}}^{t_{b}} u(\breve{l}) \Delta \breve{\iota}+\int_{t_{a}}^{t_{b}} v(\breve{l}) \Delta \breve{l}$;
b. $\int_{t_{a}}^{t_{b}}(\alpha u(\breve{l}) \Delta \breve{\iota})=\alpha \int_{t_{a}}^{t_{b}} u(\breve{\imath}) \Delta \breve{\iota}$;
c. $\int_{t_{a}}^{t_{b}} u(\breve{l}) \Delta \breve{l}=-\int_{t_{b}}^{t_{a}} u(\breve{l}) \Delta \breve{l}$;
d. $\int_{t_{a}}^{t_{b}} u(\breve{l}) \Delta \breve{\iota}=\int_{t_{a}}^{t_{c}} u(\breve{l}) \Delta \breve{\imath}+\int_{t_{c}}^{t_{b}} u(\breve{l}) \Delta \breve{l}$;
e. $\int_{t_{a}}^{t_{b}} u(\sigma(\breve{l})) v^{\Delta}(\breve{l}) \Delta \breve{l}=(u v)\left(t_{b}\right)-(u v)\left(t_{a}\right)-\int_{t_{a}}^{t_{b}} u^{\Delta}(\breve{l}) v(\breve{l}) \Delta \breve{l}$;
f. $\int_{t_{a}}^{t_{b}} u(\breve{l}) v^{\Delta}(\breve{l}) \Delta \breve{l}=(u v)\left(t_{b}\right)-(u v)\left(t_{a}\right)-\int_{t_{a}}^{t_{b}} u^{\Delta}(\breve{l}) v(\sigma(\breve{l})) \Delta \breve{l}$;
g. $\int_{t_{a}}^{t_{a}} u(\breve{l}) \Delta \breve{l}=0$
h. If $|u(\breve{l})| \leq v(\breve{l})$ on $\left[t_{a}, t_{b}\right)$, then

$$
\left|\int_{t_{a}}^{t_{b}} u(\breve{l}) \Delta \breve{\iota}\right| \leq \int_{t_{a}}^{t_{b}} v(\breve{l}) \Delta \breve{l}
$$

i. If $u(\breve{l}) \geq 0$ for all $t_{a} \leq t<t_{b}$, then $\int_{t_{a}}^{t_{b}} u(\breve{l}) \Delta \breve{\iota} \geq 0$.

Definition 2.7 ([5]) We say $t_{a}<\breve{\iota} \in \mathbb{T}^{\mathcal{K}^{k-1}}$ is a generalized zero (GZ) of order greater than or equal to $k$ of $u$ if

$$
u^{\Delta^{j}}(\breve{\iota})=0, \quad j=0,1, \ldots, k-1 .
$$

Lemma 2.8 (Rolle's theorem, [5]) If $u$ has at least $k \in \mathbb{N}$ GZs on $\mathbb{T}$, counting multiplicities, then $u^{\Delta}$ has at least $k-1$ GZs on $\mathbb{T}^{\mathcal{K}}$, counting multiplicities.

Remark 2.9 ([3]) $u^{\Delta \sigma}$ and $u^{\sigma \Delta}$ are not equal in general even if both exist which is clear from the given relation

$$
u^{\sigma \Delta}=\left(1+\mu^{\Delta}\right) u^{\Delta \sigma},
$$

although for the time scales with constant graininess functions $\mu(\breve{l})$, such as $\mathbb{T}=\mathbb{R}, \mathbb{T}=\mathbb{Z}$,

$$
u^{\Delta \sigma}=u^{\sigma \Delta} .
$$

We intensively use this fact to accomplish the key results in Sects. 3.1 and 3.3.

\subsection{Discrete calculus}

This section recalls the following basic lemma from discrete calculus which is of great interest.

Here we use these notations $\mathbb{N}=\{0,1,2, \ldots\}, \mathbb{N}\left(t_{a}\right)=\left\{t_{a}, t_{a}+1, t_{a}+2, \ldots\right\}$, where $t_{a} \in \mathbb{N}$, $\mathbb{N}\left(t_{a}, t_{b}\right)=\left\{t_{a}, t_{a}+1, t_{a}+2, \ldots, t_{b}\right\}$, where $t_{a}<t_{b}<\infty$ and $t_{a}, t_{b} \in \mathbb{N}$. 
Lemma 2.10 ([15]) Let $u(\breve{l})$ and $v(\breve{\iota})$ be defined on $\mathbb{N}\left(t_{a}\right)$. Then, for all $\breve{\iota} \in \mathbb{N}\left(t_{a}\right)$

i. Product rule:

$$
\begin{aligned}
\Delta(u(\breve{l}) v(\breve{\imath})) & =v(\breve{\imath}) \Delta u(\breve{l})+u(\breve{\iota}+1) \Delta v(\breve{l}) \\
& =u(\breve{\iota}) \Delta v(\breve{l})+v(\breve{\imath}+1) \Delta u(\breve{l}) .
\end{aligned}
$$

ii. Summation by parts:

$$
\sum_{\breve{l}=t_{a}}^{t_{b}-1} u(\breve{l}) \Delta v(\breve{l})=\left.u(\breve{l}) v(\breve{l})\right|_{t_{a}} ^{t_{b}}-\sum_{\breve{l}=t_{a}}^{t_{b}-1} \Delta u(\breve{l}) v(\breve{l}+1) .
$$

\section{3 -Convex functions}

Let $u: I \longrightarrow \mathbb{R}$, where $I$ is bounded and closed interval contained in $\mathbb{R}$, and let $a_{1}, a_{2} \in I$ then, for all $\delta \in[0,1]$, if

$$
u\left(\delta x_{1}+(1-\delta) a_{2}\right) \leq \delta u\left(x_{1}\right)+(1-\delta) u\left(a_{2}\right)
$$

holds, then $u$ is known as a convex function.

Let $x_{0}<x_{1}<\cdots<x_{n}$ be dissimilar points in $I$. An $n$th order divided difference of $u$ for these points is defined recursively as

$$
\left[x_{i}\right] u=u\left(x_{i}\right) \quad(0 \leq i \leq n) .
$$

Definition 2.11 The function $u$ is called $n$-convex in $[a, b]$ if we have

$$
\left[x_{0}, x_{1}, \ldots, x_{n}\right] u \geq 0
$$

for all $a \leq x_{0}<x_{1}<\cdots<x_{n} \leq b . u$ is called $\mathrm{n}$-concave if $-u$ is $n$-convex.

We state some basic properties of $n$-convex functions $[9,16]$. The 0 -convex function is nonnegative function, 1-convex function is simply non-decreasing and a 2-convex function is just a convex. $u$ is $n$-convex if and only if $u^{(n)} \geq 0$, provided that $u^{(n)}$ exists.

Definition 2.12 ([17]) If $\left\{a_{n}\right\}$ is an $n$-convex real sequence, $n \geq 2$, then

$$
\Delta^{n} a \geq 0,
$$

where $\Delta^{n}$ is the $n$th forward difference.

A 2-convex sequence is just a convex sequence with

$$
\Delta^{2} a_{n}=a_{n}-2 a_{n+1}+a_{n+2} \geq 0 .
$$

It is clear that, if $u:[0, \infty) \longrightarrow \mathbb{R}$ is a convex function, then $a_{n}=u(n)$ is a convex sequence. 


\section{Main results}

This section is devoted to providing our main results of this article. We are considering the time scales having constant graininess functions throughout this section.

\subsection{Higher order convexity on time scales}

We have seen in the preliminaries section that $n$-convexity is defined through the $n$th divided difference in the continuous as well as in the discrete case, so let us define the divided difference in time scales first. Let $\breve{l}_{0}, \breve{\iota}_{1}, \ldots, \breve{\iota}_{n} \in \mathbb{T}$ be $n+1$ distinct points, then consider the subset of $\mathbb{T}$ that is $\tilde{\mathbb{T}}=\left\{\breve{\iota}_{0}, \breve{\iota}_{1}, \ldots, \breve{\iota}_{n}\right\}$, where $\tilde{\mathbb{T}}$ is a closed subset of $\mathbb{R}$ which is also a time scale. Now we can write every $\breve{\iota}_{i}$ for $i=1,2, \ldots, n$ in terms of the forward value of $\breve{\iota}_{0}$,

$$
\breve{\iota}_{1}=\sigma\left(\breve{\iota}_{0}\right), \quad \breve{\iota}_{2}=\sigma\left(\sigma\left(\breve{\iota}_{0}\right)\right)=\sigma^{2}\left(\breve{\iota}_{0}\right), \quad \ldots, \quad \breve{\iota}_{n}=\sigma^{n}\left(\breve{\iota}_{0}\right) .
$$

Definition 3.1 The divided difference of the function $u: \mathbb{T} \rightarrow \mathbb{R}$, for arguments $\breve{\iota}_{0}, \breve{\iota}_{1}$ is denoted by $u\left[\breve{\iota}_{0}, \breve{l}_{1}\right]$, and defined on $\tilde{\mathbb{T}}$, by the given relation

$$
u\left[\breve{\iota}_{0}, \sigma\left(\breve{\iota}_{0}\right)\right]=\frac{u\left(\sigma\left(\breve{\iota}_{0}\right)\right)-u\left(\breve{\iota}_{0}\right)}{\sigma\left(\breve{\iota}_{0}\right)-\breve{\iota}_{0}}=\frac{u\left(\breve{\iota}_{0}\right)-u\left(\sigma\left(\breve{\iota}_{0}\right)\right)}{\breve{\iota}_{0}-\sigma\left(\breve{\iota}_{0}\right)}=u\left[\sigma\left(\breve{\iota}_{0}\right), \breve{\iota}_{0}\right] .
$$

The divided difference of three arguments $\breve{\iota}_{0}, \breve{\iota}_{1}, \breve{\iota}_{2}$ can be defined in terms of two divided differences of two arguments having a common argument, that is,

$$
u\left[\breve{\iota}_{0}, \sigma\left(\breve{\iota}_{0}\right), \sigma^{2}\left(\breve{\iota}_{0}\right)\right]=\frac{u\left[\sigma\left(\breve{\iota}_{0}\right), \sigma^{2}\left(\breve{\iota}_{0}\right)\right]-u\left[\breve{\iota}_{0}, \sigma\left(\breve{\iota}_{0}\right)\right]}{\sigma^{2}\left(\breve{\iota}_{0}\right)-\breve{\iota}_{0}} .
$$

By proceeding in the same way we can define the divided difference of $n+1$ arguments in terms of two divided differences of $n$ arguments,

$$
u\left[\breve{\iota}_{0}, \sigma\left(\breve{\iota}_{0}\right), \ldots, \sigma^{n}\left(\breve{\iota}_{0}\right)\right]=\frac{u\left[\sigma\left(\breve{\iota}_{0}\right), \sigma^{2}\left(\breve{\iota}_{0}\right), \ldots, \sigma^{n}\left(\breve{\iota}_{0}\right)\right]-u\left[\breve{t}_{0}, \sigma\left(\breve{\iota}_{0}\right), \ldots, \sigma^{n-1}\left(\breve{\iota}_{0}\right)\right]}{\sigma^{n}\left(\breve{\iota}_{0}\right)-\breve{\iota}_{0}} .
$$

Now we are able to define $n$-convex functions on time scales.

Definition 3.2 A function $u: \mathbb{T} \longrightarrow \mathbb{R}$ is called an $n$-convex function on time scales if the condition holds that

$$
u\left[\breve{\iota}_{0}, \sigma\left(\breve{\iota}_{0}\right), \ldots, \sigma^{n}\left(\breve{\iota}_{0}\right)\right] \geq 0 .
$$

For $\mathbb{T}=\mathbb{R}$ or $\mathbb{T}=h \mathbb{Z}, \tilde{\mathbb{T}}$ remains the same as $\mathbb{T}$, that is, $\tilde{\mathbb{T}}=\left\{\breve{\iota}_{0}, \sigma\left(\breve{\iota}_{0}\right), \ldots, \sigma^{n}\left(\breve{l}_{0}\right)\right\}$.

We also need to prove that, if our function satisfies the smoothness condition, that is, if $u^{\Delta^{n}}$ exists, $u$ is $n$-convex if and only if $u^{\Delta^{n}} \geq 0$, for the first condition we require the following results to establish the relation between divided difference and $\Delta$-derivative.

Remark 3.3 In [3, P-14] the following formula is given to calculate the $\Delta^{n}$-derivative of functions defined on time scales having constant graininess function $\mu(\breve{l})=c$,

$$
f^{\Delta^{n}}(\breve{\iota})=\frac{1}{c^{n}} \sum_{i=0}^{n}\left(\begin{array}{l}
n \\
i
\end{array}\right)(-1)^{i-k} f(\breve{\imath}+c k) .
$$


Let $f(\breve{\imath})=f_{1}(\breve{\imath})=a_{1} \breve{\iota}+a_{0}$ such that $\breve{\iota} \in \mathbb{T}$ and $a_{0}, a_{1} \in \mathbb{R}$, then (3.1) gets the following form for $n=1$ :

$$
\begin{aligned}
f_{1}^{\Delta}(\breve{\iota}) & =\frac{1}{c}\left\{\left(\begin{array}{l}
1 \\
0
\end{array}\right)(-1)^{1} f_{1}(\breve{\iota})+\left(\begin{array}{l}
1 \\
1
\end{array}\right)(-1)^{0} f_{1}(\breve{\imath}+c)\right\} \\
& =\frac{1}{c}\left\{-a_{1} t-a_{0}+a_{1} t+a_{1} c+a_{0}\right\}=a_{1} 1 ! .
\end{aligned}
$$

If we let $f(\breve{\iota})=f_{2}(\breve{\iota})=a_{2} \breve{\iota}^{2}+a_{1} \breve{\iota}+a_{0}$, then, for $n=2$, we get

$$
\begin{aligned}
f_{2}^{\Delta^{2}}(\breve{\iota})= & \frac{1}{c^{2}}\left\{\left(\begin{array}{l}
2 \\
0
\end{array}\right)(-1)^{2} f_{2}(\breve{\iota})+\left(\begin{array}{l}
2 \\
1
\end{array}\right)(-1)^{1} f_{2}(\breve{\imath}+c)+\left(\begin{array}{l}
2 \\
2
\end{array}\right)(-1)^{0} f_{2}(\breve{\imath}+2 c)\right\} \\
= & \frac{1}{c^{2}}\left\{a_{2} \breve{\iota}^{2}+a_{1} \breve{\iota}+a_{0}-2\left(a_{2} \breve{\iota}^{2}+2 a_{2} \breve{\iota} c+a_{2} c^{2}+a_{1} \breve{\iota}+a_{1} c+a_{0}\right)\right. \\
& \left.+a_{2} \breve{\iota}^{2}+4 a_{2} \breve{\iota} c+4 a_{2} c^{2}+a_{1} \breve{\iota}+2 a_{1} c+a_{0}\right\} \\
= & a_{2} 2 ! .
\end{aligned}
$$

Now for $n=3$, we get $f_{3}^{\Delta^{3}}(\breve{l})=a_{3} 3$ !, and so on.

In particular if we let $f(\breve{\iota})=f_{n}(\breve{\imath})=a_{n} \breve{\iota}^{n}+a_{n-1} \breve{\iota}^{n-1}+\cdots+a_{1} \breve{\iota}+a_{0}$ be a polynomial function of degree $n$ such that $\breve{\iota} \in \mathbb{T}$ and $a_{0}, a_{1}, \ldots, a_{n} \in \mathbb{R}$, then

$$
f_{n}^{\Delta^{n}}(\breve{\iota})=a_{n} n !
$$

Theorem 3.4 Let $u(\breve{l}) \in C_{\mathrm{rd}}^{n}\left(\left[t_{a}, t_{b}\right]_{\mathbb{T}}, \mathbb{R}\right)$, let $T_{n-1}(\breve{l})$ be the interpolation polynomial for $u(\breve{l})$ with respect to $n$ distinct points from $\tilde{\mathbb{T}}$, such that $\tilde{\mathbb{T}}=\left\{\sigma^{i}\left(\breve{\iota}_{0}\right) \mid \breve{\iota}_{i} \in \mathbb{T} ; i=0,1, \ldots, n-1\right\}$. Then, for each $\breve{\iota} \in\left[t_{a}, t_{b}\right]_{\mathbb{T}}$ there exists a point $\gamma=\gamma(\breve{l})$ in the open interval:

$$
\min \left(\breve{\iota}_{0}, \sigma\left(\breve{\iota}_{0}\right), \ldots, \sigma^{n-1}\left(\breve{\iota}_{0}\right), \breve{\iota}\right)=t_{a}<\gamma<t_{b}=\max \left(\breve{l}_{0}, \sigma\left(\breve{\iota}_{0}\right), \ldots, \sigma^{n-1}\left(\breve{\iota}_{0}\right), \breve{\iota}\right),
$$

such that

$$
\begin{aligned}
u(\breve{l})-T_{n-1}(\breve{l}) & \equiv R_{n-1}(\breve{l})=\frac{\left(\breve{\iota}-\breve{\iota}_{0}\right)\left(\breve{\iota}-\sigma\left(\breve{l}_{0}\right)\right) \cdots\left(\breve{\iota}-\sigma^{n-1}\left(\breve{\iota}_{0}\right)\right)}{n !} u^{\Delta^{n}}(\gamma) \\
& \equiv \frac{\psi_{n-1}(\breve{l})}{n !} u^{\Delta^{n}}(\gamma),
\end{aligned}
$$

where $\psi_{n-1}(\breve{l})=\left(\breve{l}-t_{0}\right)\left(\breve{\imath}-\sigma\left(\breve{\iota}_{0}\right)\right) \cdots\left(\breve{\iota}-\sigma^{n-1}\left(\breve{\iota}_{0}\right)\right)$.

Proof Clearly

$$
R_{n-1}\left(\breve{l}_{0}\right)=R_{n}\left(\sigma\left(\breve{l}_{0}\right)\right)=\cdots=R_{n-1}\left(\sigma^{n-1}\left(\breve{l}_{0}\right)\right)=0,
$$

we define $G_{n-1}(\breve{l})$, for any $\breve{\imath} \neq \sigma^{i}\left(\breve{\iota}_{0}\right)$, by setting

$$
R_{n-1}(\breve{l})=\left(\breve{\iota}-\breve{\iota}_{0}\right)\left(\breve{\iota}-\sigma\left(\breve{\iota}_{0}\right)\right) \cdots\left(\breve{\iota}-\sigma^{n-1}\left(\breve{\iota_{0}}\right)\right) G_{n-1}(\breve{l})=\psi_{n-1}(\breve{l}) G_{n-1}(\breve{l}) .
$$

By fixing $\breve{\imath}$ we define another function $\Omega(s)$ by

$$
\Omega(s)=u(s)-T_{n-1}(s)-\psi_{n-1}(s) G_{n-1}(\breve{l}) .
$$


Since $\Omega(s) \in C_{\mathrm{rd}}^{n}\left(\left[t_{a}, t_{b}\right]_{\mathbb{T}}, \mathbb{R}\right)$ whenever $u(s) \in C_{\mathrm{rd}}^{n}\left(\left[t_{a}, t_{b}\right]_{\mathbb{T}}, \mathbb{R}\right), \Omega^{\Delta^{n}}(s)$ is defined in $\left[t_{a}, t_{b}\right]_{\mathbb{T} \mathcal{K}^{n}}$.

We see that $\Omega(s)$ has $n+1 \mathrm{GZs}$ of order 1 in $\left[t_{a}, t_{b}\right]_{\mathbb{T}}$, namely

$$
\Omega\left(\breve{\iota}_{0}\right)=\Omega\left(\sigma\left(\breve{\iota}_{0}\right)\right)=\cdots=\Omega\left(\sigma^{n-1}\left(\breve{\iota}_{0}\right)\right)=\Omega(\breve{l})=0 .
$$

As there are $n$ adjacent intervals in $\left[t_{a}, t_{b}\right]_{\mathbb{T}}$ whose end points are GZs of $\Omega(s)$ of order 1 , by Rolle's theorem on time scales [5, Lemma 8.9], in consideration of $\Omega(s) \in C_{\mathrm{rd}}^{n}\left(\left[t_{a}, t_{b}\right]_{\mathbb{T}}, \mathbb{R}\right)$, $\Omega^{\Delta}(s)$ has $n$ GZs of order 1 in interval (3.3). They form at least $n-1$ intervals, by applying Rolle's theorem again we get $n-1$ distinct $\gamma_{i}$ in these intervals on which $\Omega^{\Delta^{2}}(s)$ has GZs. Thus, there are at least $n-1$ distinct points in the interval (3.3) at which $\Omega^{\Delta^{2}}(s)$ has GZs. Thus proceeding we get a point, say $\gamma$, in (3.3) as a GZ of the function $\Omega^{\Delta^{n}}(s)$.

On the other hand, as we know that $T_{n-1}(s)$ is an $(n-1)$ th degree polynomial function, $T_{n-1}^{\Delta^{n}}(s)=0$, and by using (3.2) we can write $\left[\psi_{n-1}(s) G_{n-1}(\breve{l})\right]^{\Delta^{n}}=1 . n ! G_{n-1}(\breve{l})$. Hence,

$$
\Omega^{\Delta^{n}}(s)=u^{\Delta^{n}}(s)-n ! G_{n-1}(\breve{l}),
$$

and since $\Omega^{\Delta^{n}}(\gamma)=0$, we obtain

$$
G_{n-1}(\breve{l})=\frac{1}{n !} u^{\Delta^{n}}(\gamma), \quad \text { for } \breve{\iota} \neq \breve{\iota}_{0}, \sigma\left(\breve{\iota}_{0}\right), \ldots, \sigma^{n-1}\left(\breve{\iota}_{0}\right) .
$$

The next result gives the representation of divided difference of $n+1$ points in terms of the $n$th delta derivative.

Theorem 3.5 Let $\left\{\breve{\iota}_{0}, \breve{\iota}_{1}, \ldots, \breve{\iota}_{n-1}\right\}=\tilde{\mathbb{T}}, \breve{\iota} \in\left[t_{a}, t_{b}\right]_{\mathbb{T}}$, be $n+1$ distinct points and let $u(z) \in$ $C_{\mathrm{rd}}^{n}\left(\left[t_{a}, t_{b}\right]_{\mathbb{T}}, \mathbb{R}\right)$, then, for some point $\gamma=\gamma(\breve{l})$ in the interval

$$
\begin{aligned}
& \min \left(\breve{\iota}, \breve{\iota}_{0}, \ldots, \sigma^{n-1}\left(\breve{\iota}_{0}\right)\right)=a<\gamma<b=\max \left(\breve{\iota}, \breve{\iota}_{0}, \ldots, \sigma^{n-1}\left(\breve{\iota}_{0}\right)\right), \\
& u\left[\breve{\iota}_{0}, \ldots, \sigma^{n-1}\left(\breve{\iota}_{0}\right), \breve{\iota}\right]=\frac{u^{\Delta^{n}}(\gamma)}{n !} .
\end{aligned}
$$

Proof By (3.9),

$$
u(\breve{l})-T_{n-1}(\breve{\iota})=\left(\breve{\iota}-\breve{\iota}_{0}\right)\left(\breve{\imath}-\sigma\left(\breve{\iota}_{0}\right)\right) \cdots\left(\breve{\iota}-\sigma^{n-1}\left(\breve{\iota}_{0}\right)\right) u\left[t_{0}, \sigma\left(\breve{l}_{0}\right), \ldots, \sigma^{n-1}\left(\breve{\iota}_{0}\right), \breve{\iota}\right]_{\mathbb{T}} .
$$

We have to use Newton's divided difference interpolation formula for $n$ distinct interpolation points from $\tilde{\mathbb{T}}$, the reader can follow the procedure given in [18, P-248],

$$
u(\breve{l})-T_{n-1}(\breve{l})=\left[\prod_{j=0}^{n-1}\left(\breve{l}-\sigma^{j}\left(\breve{\iota}_{0}\right)\right)\right] u\left[\breve{\iota}_{0}, \sigma\left(\breve{\iota}_{0}\right), \ldots, \sigma^{n-1}\left(\breve{\iota}_{0}\right), \breve{\iota}\right] .
$$

Notice that $T_{n-1}(\breve{l})$ is an interpolation polynomial which is equal to $u(\breve{l})$ at the $n$ points $\breve{\iota}_{0}, \sigma\left(\breve{\iota}_{0}\right), \ldots, \sigma^{n-1}\left(\breve{\iota}_{0}\right)$ it follows from Theorem 3.4 that

$$
\begin{aligned}
u(\breve{l})-T_{n-1}(\breve{\iota}) & \equiv R_{n-1}(\breve{\iota}) \\
& =\left(\breve{\iota}-\breve{\iota}_{0}\right)\left(\breve{\iota}-\sigma\left(\breve{\iota}_{0}\right)\right) \cdots\left(\breve{\iota}-\sigma^{n-1}\left(\breve{\iota}_{0}\right)\right) \frac{u^{n}(\gamma)}{n !} .
\end{aligned}
$$


By $\left(\breve{\iota}-t_{0}\right)\left(\breve{\iota}-\sigma\left(\breve{\iota}_{0}\right)\right) \cdots\left(\breve{\iota}-\sigma^{n-1}\left(\breve{\iota}_{0}\right)\right) \neq 0$, the desired result follows by equating the right hand-sides of (3.8) and (3.10).

From the above discussion, we immediately conclude to the following result.

Corollary 3.6 $u: \mathbb{T} \rightarrow \mathbb{R}$ is $n$-convex if and only if $u^{\Delta^{n}} \geq 0$, provided that $u^{\Delta^{n}}$ exists.

\subsection{The case of 4-convex sequence}

Theorem 3.7 Let $\vartheta(\breve{l})$ be defined on $\mathbb{N}\left[t_{a}, t_{b}\right]$ be 4-convex as well as 2-convex sequence.

Let $w: \mathbb{N}\left[t_{a}, t_{b}\right] \longrightarrow[0, \infty)$ possess 2 -concavity and satisfy

$$
w(\breve{l})=\Delta w(\breve{l})=\Delta^{2} w(\breve{\iota})=\Delta^{3} w(\breve{l})=0, \quad \breve{\iota} \in\{a, b, a+1\} .
$$

Then the following estimate is valid:

$$
\begin{aligned}
\sum_{\breve{\iota}=t_{a}}^{t_{b}-1}\left|\Delta^{2} \vartheta(\breve{\iota})\right|^{2} w(\breve{\iota}) \leq & \sum_{\breve{\iota}=t_{a}}^{t_{b}-1}\left(\frac{(\vartheta(\breve{\iota}+4))^{2}}{2}-\max |\vartheta(\breve{\iota})||u(\breve{\iota}+4)|\right) \Delta^{4} w(\breve{\iota}) \\
& +\frac{1}{2} \sum_{\breve{\iota}=t_{a}}^{t_{b}-1}(\Delta \vartheta(\breve{\iota}+3))^{2} \Delta^{3} w(\breve{\iota}) .
\end{aligned}
$$

Proof Let

$$
B=\sum_{\breve{\iota}=t_{a}}^{t_{b}-1} \Delta^{2} \vartheta(\breve{\iota}) \Delta^{2} \vartheta(\breve{l}) w(\breve{\iota})
$$

and by applying summation by parts on $B$, we get

$$
B=-\sum_{\breve{\iota}=t_{a}}^{t_{b}-1} \Delta \vartheta(\breve{\iota}+1) \Delta\left(\Delta^{2} \vartheta(\breve{\iota}) w(\breve{\iota})\right)
$$

note that here we used condition (3.11). Now we have to use the product rule for the forward difference

$$
B=-\sum_{\breve{\iota}=t_{a}}^{t_{b}-1} \Delta \vartheta(\breve{\iota}+1) \Delta^{3} \vartheta(\breve{\iota}) w(\breve{\iota})-\sum_{\breve{\iota}=t_{a}}^{t_{b}-1} \Delta \vartheta(\breve{\iota}+1) \Delta^{2} \vartheta(\breve{\iota}+1) \Delta w(\breve{\iota}) .
$$

Again by applying the summation by parts formula to the first term, we get

$$
\begin{aligned}
B= & \sum_{\breve{\iota}=t_{a}}^{t_{b}-1} \vartheta(\breve{\iota}+2) \Delta^{4} \vartheta(\breve{\iota}) w(\breve{\iota}+1)+\sum_{\breve{\iota}=t_{a}}^{t_{b}-1} \vartheta(\breve{\iota}+2) \Delta^{3} \vartheta(\breve{l}) \Delta w(\breve{\iota}) \\
& -\sum_{\breve{\iota}=t_{a}}^{t_{b}-1} \Delta \vartheta(\breve{\iota}+1) \Delta^{2} \vartheta(\breve{\iota}+1) \Delta w(\breve{\iota}) .
\end{aligned}
$$


Applying the summation by parts formula to the second term this time, we get

$$
\begin{aligned}
& B=\sum_{\breve{\iota}=t_{a}}^{t_{b}-1} \vartheta(\breve{\iota}+2) \Delta^{4} \vartheta(\breve{\iota}) w(\breve{\imath}+1)-\sum_{\breve{\iota}=t_{a}}^{t_{b}-1} \Delta \vartheta(\breve{\imath}+2) \Delta^{2} \vartheta(\breve{\iota}+1) \Delta w(\breve{\iota}) \\
& -\sum_{\breve{\iota}=t_{a}}^{t_{b}-1} \vartheta(\breve{\iota}+3) \Delta^{2} \vartheta(\breve{l}+1) \Delta^{2} w(\breve{l})-\sum_{\breve{l}=t_{a}}^{t_{b}-1} \Delta \vartheta(\breve{l}+1) \Delta^{2} \vartheta(\breve{l}+1) \Delta w(\breve{l}) \\
& =\sum_{\breve{\iota}=t_{a}}^{t_{b}-1} \vartheta(\breve{\iota}+2) \Delta^{4} \vartheta(\breve{\iota}) w(\breve{\iota}+1) \\
& -\sum_{\breve{l}=t_{a}}^{t_{b}-1}\left(\Delta \vartheta(\breve{\iota}+2) \Delta^{2} \vartheta(\breve{\iota}+1)+\vartheta(\breve{\imath}+1) \Delta^{2} \vartheta(\breve{\imath}+1)\right) \Delta w(\breve{\iota}) \\
& -\sum_{\breve{l}=t_{a}}^{t_{b}-1} \vartheta(\breve{\imath}+3) \Delta^{2} \vartheta(\breve{\imath}+1) \Delta^{2} w(\breve{\imath}) \\
& =\sum_{\breve{l}=t_{a}}^{t_{b}-1} \vartheta(\breve{\iota}+2) \Delta^{4} \vartheta(\breve{l}) w(\breve{\iota}+1)-\sum_{\breve{l}=t_{a}}^{t_{b}-1} \Delta(\Delta \vartheta(\breve{\iota}+1))^{2} \Delta w(\breve{l}) \\
& -\sum_{\breve{\iota}=t_{a}}^{t_{b}-1} \vartheta(\breve{\imath}+3) \Delta^{2} \vartheta(\breve{\imath}+1) \Delta^{2} w(\breve{l})
\end{aligned}
$$

By applying the summation by parts formula to the second term twice, we get

$$
\begin{aligned}
& B=\sum_{\breve{l}=t_{a}}^{t_{b}-1} \vartheta(\breve{\iota}+2) \Delta^{4} \vartheta(\breve{l}) w(\breve{\imath}+1)-\sum_{\breve{\iota}=t_{a}}^{t_{b}-1} \vartheta(\breve{\iota}+3) \Delta^{2} \vartheta(\breve{l}+2) \Delta^{2} w(\breve{l}) \\
& -\sum_{\breve{\iota}=t_{a}}^{t_{b}-1} \vartheta(\breve{\iota}+3) \Delta \vartheta(\breve{\imath}+3) \Delta^{3} w(\breve{l})-\sum_{\breve{\iota}=t_{a}}^{t_{b}-1} \vartheta(\breve{l}+3) \Delta^{2} \vartheta(\breve{l}+1) \Delta^{2} w(\breve{l}), \\
& =\sum_{\breve{l}=t_{a}}^{t_{b}-1} \vartheta(\breve{\iota}+2) \Delta^{4} \vartheta(\breve{l}) w(\breve{\iota}+1)-\sum_{\breve{\iota}=t_{a}}^{t_{b}-1} \vartheta(\breve{\iota}+3)\left(\Delta^{2} \vartheta(\breve{\iota}+2)+\Delta^{2} \vartheta(\breve{l}+1)\right) \Delta^{2} w(\breve{l}) \\
& -\sum_{\breve{l}=t_{a}}^{t_{b}-1} \vartheta(\breve{\imath}+3) \Delta \vartheta(\breve{l}+3) \Delta^{3} w(\breve{l})
\end{aligned}
$$

now by breaking up the last term and adding and subtracting the term $\frac{1}{2} \sum_{\breve{l}=t_{a}}^{t_{b}-1} \vartheta(\breve{\imath}+$ 4) $\Delta u(\breve{\iota}+3) \Delta^{3} w(\breve{\imath})$, we get

$$
\begin{aligned}
& B=\sum_{\breve{\iota}=t_{a}}^{t_{b}-1} \vartheta(\breve{\iota}+2) \Delta^{4} \vartheta(\breve{\imath}) w(\breve{\iota}+1)-\sum_{\breve{\iota}=t_{a}}^{t_{b}-1} \vartheta(\breve{\imath}+3)\left(\Delta^{2} \vartheta(\breve{\imath}+2)+\Delta^{2} \vartheta(\breve{\imath}+1)\right) \Delta^{2} w(\breve{\iota}) \\
& -\frac{1}{2} \sum_{\breve{l}=t_{a}}^{t_{b}-1}(\vartheta(\breve{\imath}+3) \Delta \vartheta(\breve{\imath}+3)+\vartheta(\breve{\imath}+4) \Delta \vartheta(\breve{\imath}+3)) \Delta^{3} w(\breve{l}) \\
& +\frac{1}{2} \sum_{\breve{l}=t_{a}}^{t_{b}-1} \Delta \vartheta(\breve{\iota}+3)(\vartheta(\breve{l}+4)-\vartheta(\breve{\imath}+3)) \Delta^{3} w(\breve{l}) .
\end{aligned}
$$


By using the third term with the difference of $(\vartheta(\breve{l}+3))^{2}$, and the last term with the difference of $\vartheta(\breve{l}+3)$ and applying the summation by parts formula to the third term, we get

$$
\begin{aligned}
B= & \sum_{\breve{\iota}=t_{a}}^{t_{b}-1} \vartheta(\breve{\iota}+2) \Delta^{4} \vartheta(\breve{l}) w(\breve{\imath}+1)-\sum_{\breve{\iota}=t_{a}}^{t_{b}-1} \vartheta(\breve{\iota}+3)\left(\Delta^{2} \vartheta(\breve{l}+2)+\Delta^{2} \vartheta(\breve{l}+1)\right) \Delta^{2} w(\breve{l}) \\
& +\frac{1}{2} \sum_{\breve{l}=t_{a}}^{t_{b}-1}(\vartheta(\breve{l}+4))^{2} \Delta^{4} w(\breve{l})+\frac{1}{2} \sum_{\breve{l}=t_{a}}^{t_{b}-1} \Delta \vartheta(\breve{\iota}+3)(\Delta \vartheta(\breve{\iota}+3)) \Delta^{3} w(\breve{l}) .
\end{aligned}
$$

We can write

$$
\begin{aligned}
& B \leq \max _{t \in\left[t_{a}, t_{b}\right] \mathbb{Z}}|\vartheta(\breve{l})| \sum_{\breve{\imath}=t_{a}}^{t_{b}-1}\left|\Delta^{4} \vartheta(\breve{l})\right| w(\breve{l}+1) \\
& +\max _{t \in\left[t_{a}, t_{b}\right] \mathbb{Z}}|\vartheta(\breve{l})| \sum_{\breve{l}=t_{a}}^{t_{b}-1}\left|\Delta^{2} \vartheta(\breve{l}+2)+\Delta^{2} \vartheta(\breve{l}+1)\right|\left|\Delta^{2} w(\breve{l})\right| \\
& +\frac{1}{2} \sum_{\breve{l}=t_{a}}^{t_{b}-1}(\vartheta(\breve{\imath}+4))^{2} \Delta^{4} w(\breve{\imath})+\frac{1}{2} \sum_{\breve{\iota}=t_{a}}^{t_{b}-1} \Delta \vartheta(\breve{\iota}+3)(\Delta \vartheta(\breve{\imath}+3)) \Delta^{3} w(\breve{\imath}) .
\end{aligned}
$$

Now by using 2 - as well as 4-convexity of $\vartheta(\breve{l})$ and concavity of the weight function, we get

$$
\begin{aligned}
& B \leq \max _{t \in\left[t_{a}, t_{b}\right]_{\mathbb{Z}}}|\vartheta(\breve{\iota})| \sum_{\breve{\iota}=t_{a}}^{t_{b}-1} \Delta^{4} \vartheta(\breve{l}) w(\breve{l}+1) \\
& -\max _{t \in\left[t_{a}, t_{b}\right]_{\mathbb{Z}}}|\vartheta(\breve{l})| \sum_{\breve{\iota}=t_{a}}^{t_{b}-1}\left(\Delta^{2} \vartheta(\breve{\imath}+2)+\Delta^{2} \vartheta(\breve{l}+1)\right) \Delta^{2} w(\breve{l}) \\
& +\frac{1}{2} \sum_{\breve{l}=t_{a}}^{t_{b}-1}(\vartheta(\breve{l}+4))^{2} \Delta^{4} w(\breve{\iota})+\frac{1}{2} \sum_{\breve{l}=t_{a}}^{t_{b}-1}(\Delta \vartheta(\breve{l}+3))^{2} \Delta^{3} w(\breve{\iota}) .
\end{aligned}
$$

Finally by using the summation by parts formula four times on the first term and two times on the second term, respectively, we get

$$
\begin{aligned}
B \leq & \max _{\breve{l} \in\left[t_{a}, t_{b}\right]_{\mathbb{Z}}}|\vartheta(\breve{l})| \sum_{\breve{l}=t_{a}}^{t_{b}-1} \vartheta(\breve{\iota}+3) \Delta^{4} w(\breve{l})-\max _{\breve{\iota} \in\left[t_{a}, t_{b}\right]_{\mathbb{Z}}}|\vartheta(\breve{l})| \sum_{\breve{\iota}=t_{a}}^{t_{b}-1}(\vartheta(\breve{\iota}+4)+\vartheta(\breve{\iota}+3)) \Delta^{4} w(\breve{l}) \\
& +\frac{1}{2} \sum_{\breve{l}=t_{a}}^{t_{b}-1}(\vartheta(\breve{\imath}+4))^{2} \Delta^{4} w(\breve{l})+\frac{1}{2} \sum_{\breve{l}=t_{a}}^{t_{b}-1}(\Delta \vartheta(\breve{l}+3))^{2} \Delta^{3} w(\breve{l}),
\end{aligned}
$$

from which follows the desired inequality.

The next result describes the energy estimates for the difference of two 4-convex sequences defined on $\mathbb{N}\left[t_{a}, t_{b}\right]$. 
Corollary 3.8 Let $\vartheta_{1}, \vartheta_{2}$ be 4-convex as well as 2-convex sequences defined on $\mathbb{N}\left[t_{a}, t_{b}\right]$, and let $w$ satisfy the conditions of Theorem 3.7. Then the following estimate is valid:

$$
\begin{aligned}
& \sum_{\breve{l}=t_{a}}^{t_{b}-1}\left|\Delta^{2} \vartheta_{2}(\breve{l})-\Delta^{2} \vartheta_{1}(\breve{l})\right|^{2} w(\breve{l}) \\
& \leq \sum_{\breve{l}=t_{a}}^{t_{b}-1}\left(\frac{\left(\vartheta_{2}(\breve{l}+4)-\vartheta_{1}(\breve{\imath}+4)\right)^{2}}{2}-\max \left|\vartheta_{2}(\breve{l})-\vartheta_{1}(\breve{l})\right|\left[\vartheta_{2}(\breve{l}+4)+\vartheta_{1}(\breve{\imath}+4)\right]\right) \Delta^{4} w(\breve{l}) \\
& +\frac{1}{2} \sum_{\breve{l}=t_{a}}^{t_{b}-1}\left(\Delta \vartheta_{2}(\breve{l}+3)-\Delta \vartheta_{1}(\breve{l}+3)\right)^{2} w^{\Delta^{3}}(\breve{l}) .
\end{aligned}
$$

Proof By letting $\vartheta(\breve{l})=\vartheta_{2}(\breve{l})-\vartheta_{1}(\breve{l})$ we can write

$$
\left|\Delta^{2} \vartheta_{2}(\breve{l})-\Delta^{2} \vartheta_{1}(\breve{l})\right| \leq\left|\Delta^{2} \vartheta_{2}(\breve{l})\right|+\left|\Delta^{2} \vartheta_{1}(\breve{l})\right|=\Delta^{2} \vartheta_{1}(\breve{l})+\Delta^{2} \vartheta_{2}(\breve{l})
$$

similarly

$$
\left|\Delta^{4} \vartheta_{2}(\breve{l})-\Delta^{4} \vartheta_{1}(\breve{l})\right| \leq\left|\Delta^{4} \vartheta_{2}(\breve{l})\right|+\left|\Delta^{4} \vartheta_{1}(\breve{l})\right|=\Delta^{4} \vartheta_{1}(\breve{l})+\Delta^{4} \vartheta_{2}(\breve{l})
$$

then, by applying Theorem 3.7, we get

$$
\begin{aligned}
& \sum_{\breve{l}=t_{a}}^{t_{b}-1}\left|\Delta^{2} \vartheta_{2}(\breve{l})-\Delta^{2} \vartheta_{1}(\breve{l})\right|^{2} w(\breve{l}) \\
& \leq \sum_{\breve{l}=t_{a}}^{t_{b}-1}\left(\frac{\left(\vartheta_{2}(\breve{l}+4)-\vartheta_{1}(\breve{\imath}+4)\right)^{2}}{2}-\max \left|\vartheta_{2}(\breve{l})-\vartheta_{1}(\breve{l})\right|\left[\vartheta_{2}(\breve{l}+4)+\vartheta_{1}(\breve{\imath}+4)\right]\right) \Delta^{4} w(\breve{l}) \\
& +\frac{1}{2} \sum_{\breve{l}=t_{a}}^{t_{b}-1}\left(\Delta \vartheta_{2}(\breve{l}+3)-\Delta \vartheta_{1}(\breve{l}+3)\right)^{2} w^{\Delta^{3}}(\breve{l}) .
\end{aligned}
$$

This leads us towards the following remark.

Remark 3.9 Let $\vartheta_{1}, \vartheta_{2}$ and $w(\breve{l})$ be similar in the earlier result. By applying the discrete Hölder inequality, we obtain

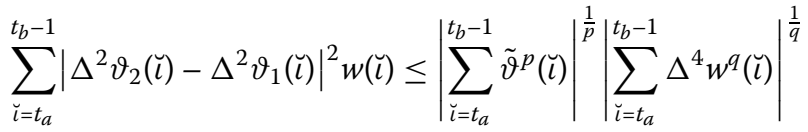

$$
\begin{aligned}
& +\frac{1}{2} \sum_{\breve{l}=t_{a}}^{t_{b}-1}\left(\Delta \vartheta_{2}(\breve{\iota}+3)-\Delta \vartheta_{1}(\breve{l}+3)\right)^{2} w^{\Delta^{3}}(\breve{l}),
\end{aligned}
$$

where

$$
\tilde{\vartheta}(\breve{l})=\frac{\left(\vartheta_{2}(\breve{\iota}+4)-\vartheta_{1}(\breve{\iota}+4)\right)^{2}}{2}-\max \left|\vartheta_{2}(\breve{\iota})-\vartheta_{1}(\breve{\iota})\right|\left[\vartheta_{2}(\breve{l}+4)+\vartheta_{1}(\breve{\iota}+4)\right] .
$$




\subsection{The case of 4-convex functions on time scales}

The subsequent results are of great significance, they unify the results presented in Sect. 3.2 and the results established in [1], by using the tools of time scale calculus.

Theorem 3.10 Let $u(\breve{l}) \in C_{\mathrm{rd}}^{4}\left(\left[t_{a}, t_{b}\right]_{\mathbb{T}}, \mathbb{R}\right)$ be 4-convex as well as 2-convex, let $w$ be a nonnegative 2-concave function in $C_{\mathrm{rd}}^{4}\left[t_{a}, t_{b}\right]_{\mathbb{T}}$, satisfying

$$
w(\breve{l})=w^{\Delta}(\breve{l})=w^{\Delta^{2}}(\breve{l})=w^{\Delta^{3}}(\breve{l})=0, \quad t \in\{a, b, \sigma(a)\} .
$$

Then the following estimate is true:

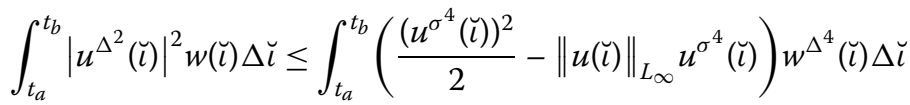

$$
\begin{aligned}
& +\frac{\mu(\breve{l})}{2} \int_{t_{a}}^{t_{b}}\left(u^{\Delta \sigma^{3}}(\breve{\iota})\right)^{2} w^{\Delta^{3}}(\breve{\iota}) \Delta \breve{\iota} .
\end{aligned}
$$

Proof Consider

$$
A=\int_{t_{a}}^{t_{b}}\left[u^{\Delta^{2}}(\breve{l})\right]^{2} w(\breve{l}) \Delta \breve{\iota} .
$$

This can be written as

$$
A=\int_{t_{a}}^{t_{b}} u^{\Delta^{2}}(\breve{l})\left(u^{\Delta^{2}}(\breve{\iota}) w(\breve{\iota})\right) \Delta \breve{\iota}
$$

now applying the $\Delta$-integration by parts formula, Theorem 2.6 , we get

$$
\begin{aligned}
A & =-\int_{t_{a}}^{t_{b}} u^{\Delta \sigma}(\breve{l})\left(u^{\Delta^{2}}(\breve{\iota}) w(\breve{l})\right)^{\Delta} \Delta \breve{\iota} \\
& =-\int_{t_{a}}^{t_{b}} u^{\Delta \sigma}(\breve{l}) u^{\Delta^{3}}(\breve{\iota}) w(\breve{\imath}) \Delta \breve{\iota}-\int_{t_{a}}^{t_{b}} u^{\Delta \sigma}(\breve{l}) u^{\Delta^{2} \sigma}(\breve{l}) w^{\Delta}(\breve{\iota}) \Delta \breve{\iota} .
\end{aligned}
$$

Here we used condition (3.15) on the weight function $w$. By applying (2.1) and $\Delta$ integration by parts to the first integral again, see Theorem 2.6, we get

$$
\begin{aligned}
A= & \int_{t_{a}}^{t_{b}} u^{\sigma \sigma}(\breve{l}) u^{\Delta^{3}}(\breve{l}) w^{\Delta}(\breve{\imath}) \Delta \breve{\iota}+\int_{t_{a}}^{t_{b}} u^{\sigma \sigma}(\breve{l}) u^{\Delta^{4}}(\breve{l}) w^{\sigma}(\breve{\imath}) \Delta \breve{\iota} \\
& -\int_{t_{a}}^{t_{b}} u^{\Delta \sigma}(\breve{l}) u^{\Delta^{2} \sigma}(\breve{\iota}) w^{\Delta}(\breve{\imath}) \Delta \breve{\iota} .
\end{aligned}
$$

By applying (2.1) and the $\Delta$-integration by parts formula to the first integral we get

$$
\begin{aligned}
A= & \int_{t_{a}}^{t_{b}} u^{\sigma \sigma}(\breve{\iota}) u^{\Delta^{4}}(\breve{\iota}) w^{\sigma}(\breve{\iota}) \Delta \breve{\iota}-\int_{t_{a}}^{t_{b}}\left[u^{\Delta \sigma}(\breve{l}) u^{\Delta^{2} \sigma}(\breve{\iota})+u^{\Delta \sigma \sigma}(\breve{l}) u^{\Delta^{2} \sigma}(\breve{l})\right] w^{\Delta}(\breve{l}) \Delta \breve{\iota} \\
& -\int_{t_{a}}^{t_{b}} u^{\sigma^{3}}(\breve{\imath}) u^{\Delta^{2} \sigma}(\breve{\imath}) w^{\Delta^{2}}(\breve{\imath}) \Delta \breve{\iota}
\end{aligned}
$$




$$
\begin{aligned}
= & \int_{t_{a}}^{t_{b}} u^{\sigma \sigma}(\breve{\imath}) u^{\Delta^{4}}(\breve{\imath}) w^{\sigma}(\breve{\imath}) \Delta \breve{\iota}-\int_{t_{a}}^{t_{b}}\left[\left(u^{\Delta \sigma}(\breve{l})\right)^{2}\right]^{\Delta} w^{\Delta}(\breve{\iota}) \Delta \breve{\iota} \\
& -\int_{t_{a}}^{t_{b}} u^{\sigma^{3}}(\breve{\imath}) u^{\Delta^{2} \sigma}(\breve{\imath}) w^{\Delta^{2}}(\breve{\imath}) \Delta \breve{l} .
\end{aligned}
$$

Now by applying the $\Delta$-integration by parts formula to the second integral we get

$$
\begin{aligned}
& A=\int_{t_{a}}^{t_{b}} u^{\sigma \sigma}(\breve{l}) u^{\Delta^{4}}(\breve{l}) w^{\sigma}(\breve{l}) \Delta \breve{\iota}+\int_{t_{a}}^{t_{b}}\left(u^{\Delta \sigma \sigma}(\breve{\iota})\right)^{2} w^{\Delta \Delta}(\breve{l}) \Delta \breve{\iota}-\int_{t_{a}}^{t_{b}} u^{\sigma^{3}}(\breve{\imath}) u^{\Delta^{2} \sigma}(\breve{l}) w^{\Delta^{2}}(\breve{\iota}) \Delta \breve{\iota}, \\
& A=\int_{t_{a}}^{t_{b}} u^{\sigma \sigma}(\breve{\iota}) u^{\Delta^{4}}(\breve{\iota}) w^{\sigma}(\breve{l}) \Delta \breve{\iota}-\int_{t_{a}}^{t_{b}} u^{\sigma^{3}}(\breve{\iota})\left[u^{\Delta^{2} \sigma \sigma}(\breve{l})+u^{\Delta^{2} \sigma}(\breve{l})\right] w^{\Delta^{2}}(\breve{l}) \Delta \breve{\iota} \\
& -\int_{t_{a}}^{t_{b}} u^{\sigma^{3}}(\breve{l}) u^{\Delta \sigma^{3}}(\breve{l}) w^{\Delta^{3}}(\breve{l}) \Delta \breve{l},
\end{aligned}
$$

now by breaking up the last term and adding and subtracting the term $\frac{1}{2} \int_{t_{a}}^{t_{b}} u^{\sigma^{4}}(\breve{l}) u^{\Delta \sigma^{3}}(\breve{l}) \times$ $w^{\Delta^{3}}(\breve{\iota}) \Delta \breve{\iota}$, we get

$$
\begin{aligned}
& A=\int_{t_{a}}^{t_{b}} u^{\sigma \sigma}(\breve{l}) u^{\Delta^{4}}(\breve{l}) w^{\sigma}(\breve{l}) \Delta \breve{\iota}-\int_{t_{a}}^{t_{b}} u^{\sigma^{3}}(\breve{l})\left[u^{\Delta^{2} \sigma \sigma}(\breve{l})+u^{\Delta^{2} \sigma}(\breve{l})\right] w^{\Delta^{2}}(\breve{l}) \Delta \breve{\iota} \\
& -\frac{1}{2} \int_{t_{a}}^{t_{b}}\left[u^{\sigma^{3}}(\breve{l}) u^{\Delta \sigma^{3}}(\breve{\iota})+u^{\sigma^{4}}(\breve{l}) u^{\Delta \sigma^{3}}(\breve{l})\right] w^{\Delta^{3}}(\breve{l}) \Delta \breve{\iota} \\
& +\frac{1}{2} \int_{t_{a}}^{t_{b}} u^{\Delta \sigma^{3}}(\breve{\iota}) u^{\sigma^{4}}(\breve{\iota}) w^{\Delta^{3}}(\breve{l}) \Delta \breve{\iota}-\frac{1}{2} \int_{t_{a}}^{t_{b}} u^{\Delta \sigma^{3}}(\breve{\iota}) u^{\sigma^{3}}(\breve{l}) w^{\Delta^{3}}(\breve{\iota}) \Delta \breve{\iota} \text {. }
\end{aligned}
$$

By using the third term with the $\Delta$-derivative of $\left(u^{\sigma^{3}}\right)^{2}$, and applying the $\Delta$-integration by parts formula to it, we get

$$
\begin{aligned}
& A=\int_{t_{a}}^{t_{b}} u^{\sigma \sigma}(\breve{l}) u^{\Delta^{4}}(\breve{l}) w^{\sigma}(\breve{l}) \Delta \breve{\iota}-\int_{t_{a}}^{t_{b}} u^{\sigma^{3}}(\breve{l})\left[u^{\Delta^{2} \sigma \sigma}(\breve{l})+u^{\Delta^{2} \sigma}(\breve{l})\right] w^{\Delta^{2}}(\breve{l}) \Delta \breve{\iota} \\
& +\frac{1}{2} \int_{t_{a}}^{t_{b}}\left(u^{\sigma^{4}}(\breve{\iota})\right)^{2} w^{\Delta^{4}}(\breve{\iota}) \Delta \breve{\iota}+\frac{1}{2} \int_{t_{a}}^{t_{b}} u^{\Delta \sigma^{3}}(\breve{l})\left[u^{\sigma^{4}}(\breve{\iota})-u^{\sigma^{3}}(\breve{l})\right] w^{\Delta^{3}}(\breve{l}) \Delta \breve{\iota} .
\end{aligned}
$$

Now by using Theorem 2.2(d) we can write

$$
\begin{aligned}
A= & \int_{t_{a}}^{t_{b}} u^{\sigma \sigma}(\breve{\iota}) u^{\Delta^{4}}(\breve{\iota}) w^{\sigma}(\breve{l}) \Delta \breve{\iota}-\int_{t_{a}}^{t_{b}} u^{\sigma^{3}}(\breve{\iota})\left[u^{\Delta^{2} \sigma \sigma}(\breve{\iota})+u^{\Delta^{2} \sigma}(\breve{l})\right] w^{\Delta^{2}}(\breve{\imath}) \Delta \breve{\iota} \\
& +\frac{1}{2} \int_{t_{a}}^{t_{b}}\left(u^{\sigma^{4}}(\breve{\iota})\right)^{2} w^{\Delta^{4}}(\breve{\iota}) \Delta \breve{\iota}+\frac{1}{2} \int_{t_{a}}^{t_{b}} u^{\Delta \sigma^{3}}(\breve{\iota})\left[\mu^{\sigma^{3}}(\breve{\iota}) u^{\Delta \sigma^{3}}(\breve{l})\right] w^{\Delta^{3}}(\breve{\iota}) \Delta \breve{\iota} .
\end{aligned}
$$

We are considering the case of constant graininess functions only, so $\mu^{\sigma^{3}}(\breve{l})=\mu(\breve{l})$ for all $\breve{\iota} \in\left[t_{a}, t_{b}\right]_{\mathbb{T}}$, and we have

$$
\begin{aligned}
& \int_{t_{a}}^{t_{b}}\left|u^{\Delta^{2}}(\breve{\iota})\right|^{2} w(\breve{\iota}) \Delta \breve{\iota} \\
& \quad \leq \frac{1}{2} \int_{t_{a}}^{t_{b}}\left(u^{\sigma^{4}}(\breve{l})\right)^{2} w^{\Delta^{4}}(\breve{l}) \Delta \breve{\iota}+\sup _{t \in\left[t_{a}, t_{b}\right]_{\mathbb{T}}}|u(\breve{l})| \int_{t_{a}}^{t_{b}}\left|u^{\Delta^{4}}(\breve{l})\right| w^{\sigma}(\breve{l}) \Delta \breve{\iota}
\end{aligned}
$$




$$
\begin{aligned}
& +\sup _{t \in\left[t_{a}, t_{b}\right]_{\mathbb{T}}}|u(\breve{\iota})| \int_{t_{a}}^{t_{b}}\left(\left|u^{\Delta^{2} \sigma \sigma}(\breve{\iota})\right|+\left|u^{\Delta^{2} \sigma}(\breve{\iota})\right|\right)\left|w^{\Delta^{2}}(\breve{\iota})\right| \Delta \breve{\iota} \\
& +\frac{\mu(\breve{l})}{2} \int_{t_{a}}^{t_{b}}\left(u^{\Delta \sigma^{3}}(\breve{\iota})\right)^{2} w^{\Delta^{3}}(\breve{\iota}) \Delta \breve{\iota} .
\end{aligned}
$$

By using 2-convexity and 4-convexity of $u$ and the concavity of the weight function $w$, the above inequality can get the form

$$
\begin{aligned}
& \int_{t_{a}}^{t_{b}}\left|u^{\Delta^{2}}(\breve{\iota})\right|^{2} w(\breve{\imath}) \Delta \breve{\iota} \\
& \leq \frac{1}{2} \int_{t_{a}}^{t_{b}}\left(u^{\sigma^{4}}(\breve{l})\right)^{2} w^{\Delta^{4}}(\breve{l}) \Delta \breve{l}+\sup _{t \in\left[t_{a}, t_{b}\right]_{\mathbb{T}}}|u(\breve{l})| \int_{t_{a}}^{t_{b}} u^{\Delta^{4}}(\breve{l}) w^{\sigma}(\breve{l}) \Delta \breve{\iota} \\
& -\sup _{t \in\left[t_{a}, t_{b}\right]_{\mathbb{T}}}|u(\breve{l})| \int_{t_{a}}^{t_{b}}\left(u^{\Delta^{2} \sigma \sigma}(\breve{l})+u^{\Delta^{2} \sigma}(\breve{l})\right) w^{\Delta^{2}}(\breve{l}) \Delta \breve{l} \\
& +\frac{\mu(\breve{l})}{2} \int_{t_{a}}^{t_{b}}\left(u^{\Delta \sigma^{3}}(\breve{\iota})\right)^{2} w^{\Delta^{3}}(\breve{\iota}) \Delta \breve{\iota} .
\end{aligned}
$$

Finally by integrating the second integral four times and the third integral two times on the right hand side, by applying the $\Delta$-integration by parts formula and (2.1), we get

$$
\begin{aligned}
& \int_{t_{a}}^{t_{b}}\left|u^{\Delta^{2}}(\breve{\iota})\right|^{2} w(\breve{\iota}) \Delta \breve{\iota} \\
& \leq \frac{1}{2} \int_{t_{a}}^{t_{b}}\left(u^{\sigma^{4}}(\breve{l})\right)^{2} w^{\Delta^{4}}(\breve{l}) \Delta \breve{\iota}+\sup _{t \in\left[t_{a}, t_{b}\right]_{\mathbb{T}}}|u(\breve{l})| \int_{t_{a}}^{t_{b}} u^{\sigma^{3}}(\breve{l}) w^{\Delta^{4}}(\breve{\iota}) \Delta \breve{\iota} \\
& -\sup _{t \in\left[t_{a}, t_{b}\right]_{\mathbb{T}}}|u(\breve{l})| \int_{t_{a}}^{t_{b}}\left(u^{\sigma^{4}}(\breve{\iota})+u^{\sigma^{3}}(\breve{\iota})\right) w^{\Delta^{4}}(\breve{l}) \Delta \breve{\iota} \\
& +\frac{\mu^{\sigma^{3}}(\breve{l})}{2} \int_{t_{a}}^{t_{b}}\left|u^{\Delta \sigma^{3}}(\breve{\iota})\right|^{2}\left|w^{\Delta^{3}}(\breve{\iota})\right| \Delta \breve{\iota},
\end{aligned}
$$

from which follows the desired inequality (3.16).

The succeeding result represents the energy estimates for the difference of two 4-convex functions on time scales.

Corollary 3.11 Let $u_{1}, u_{2} \in C_{\mathrm{rd}}^{4}\left(\left[t_{a}, t_{b}\right]_{\mathbb{T}}, \mathbb{R}\right)$ be smooth 4 -convex as well as 2-convex, and let $w$ satisfy the conditions of Theorem 3.10. Then the following estimate is valid:

$$
\begin{aligned}
& \int_{t_{a}}^{t_{b}}\left|u_{2}^{\Delta^{2}}(\breve{\iota})-u_{1}^{\Delta^{2}}(\breve{\iota})\right|^{2} w(\breve{\iota}) \Delta \breve{\iota}
\end{aligned}
$$

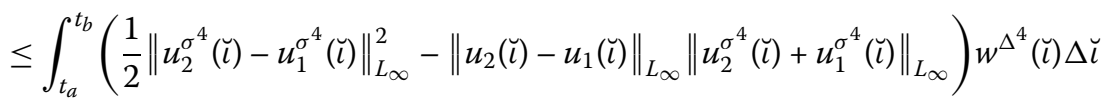

$$
\begin{aligned}
& +\frac{\mu(\breve{l})}{2} \int_{t_{a}}^{t_{b}}\left(u_{2}^{\Delta \sigma^{3}}(\breve{l})-u_{1}^{\Delta \sigma^{3}}(\breve{l})\right)^{2} w^{\Delta^{3}}(\breve{l}) \Delta \breve{\iota} .
\end{aligned}
$$


Proof Assume $u(\breve{l})=u_{2}(\breve{l})-u_{1}(\breve{l})$ then we can prove a result similar to Theorem 3.10, but the last term will get the form, through (3.19),

$$
\begin{aligned}
& \frac{1}{2} \int_{t_{a}}^{t_{b}}\left(u_{2}^{\Delta \sigma^{3}}(\breve{\iota})-u_{1}^{\Delta \sigma^{3}}(\breve{l})\right)\left[\left(u_{2}^{\sigma^{4}}(\breve{l})-u_{1}^{\sigma^{4}}(\breve{l})\right)-\left(u_{2}^{\sigma^{3}}(\breve{l})-u_{1}^{\sigma^{3}}(\breve{l})\right)\right] w^{\Delta^{3}}(\breve{l}) \Delta \breve{\iota} \\
& \quad=\frac{1}{2} \int_{t_{a}}^{t_{b}}\left(u_{2}^{\Delta \sigma^{3}}(\breve{l})-u_{1}^{\Delta \sigma^{3}}(\breve{l})\right)\left[\mu(\breve{l}) u_{2}^{\Delta \sigma^{3}}(\breve{\iota})-\mu(\breve{l}) u_{1}^{\Delta \sigma^{3}}(\breve{l})\right] w^{\Delta^{3}}(\breve{l}) \Delta \breve{\iota} \\
& \quad=\frac{\mu(\breve{l})}{2} \int_{t_{a}}^{t_{b}}\left(u_{2}^{\Delta \sigma^{3}}(\breve{\iota})-u_{1}^{\Delta \sigma^{3}}(\breve{l})\right)^{2} w^{\Delta^{3}}(\breve{\iota}) \Delta \breve{l} .
\end{aligned}
$$

We sum up this section with the following remark.

Remark 3.12 Let $u_{1}, u_{2} \in C_{\mathrm{rd}}^{4}\left(\left[t_{a}, t_{b}\right]_{\mathbb{T}}, \mathbb{R}\right)$ and $w(\breve{l})$ be similar in the earlier result. By applying Hölder's inequality on time scale [13], our estimate will get the following form:

$$
\begin{aligned}
& \int_{t_{a}}^{t_{b}}\left|u_{2}^{\Delta^{2}}(\breve{\iota})-u_{1}^{\Delta^{2}}(\breve{l})\right|^{2} w(\breve{\iota}) \Delta \breve{\iota} \\
& \leq\|\tilde{u}(\breve{l})\|_{L_{p}}\left\|w^{\Delta^{4}}(\breve{l})\right\|_{L_{q}}+\frac{\mu(\breve{l})}{2} \int_{t_{a}}^{t_{b}}\left(u_{2}^{\Delta \sigma^{3}}(\breve{l})-u_{1}^{\Delta \sigma^{3}}(\breve{l})\right)^{2} w^{\Delta^{3}}(\breve{l}) \Delta \breve{l},
\end{aligned}
$$

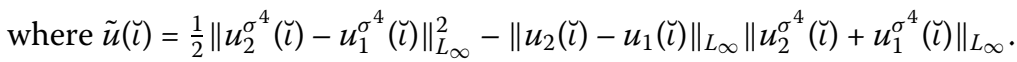

\section{Acknowledgements}

Not applicable.

\section{Funding}

The authors acknowledge the financial and moral support by the Higher Education Commission (HEC), Pakistan, via the funding of Indigenous Scholarship phase I, batch V.

\section{Availability of data and materials}

Not applicable.

\section{Competing interests}

It is asserted that no competing interests exist related to this manuscript.

\section{Authors' contributions}

The authors have equally made contributions to this manuscript. Both authors have checked the manuscript and approved the final version.

\section{Publisher's Note}

Springer Nature remains neutral with regard to jurisdictional claims in published maps and institutional affiliations.

Received: 19 September 2019 Accepted: 5 June 2020 Published online: 16 June 2020

\section{References}

1. Pečarić, J., Shoaib Saleem, M., Ahmed, I., Ahmed, N.: The weighted integral inequalities for the second derivative of 4-convex function. J. Math. Inequal. 11(2), 543-550 (2017)

2. Aulbach, B., Hilger, S.: A unified approach to continuous and discrete dynamics. Preprint, Inst. für Mathematik (1988)

3. Bohner, M., Peterson, A.: Dynamic Equations on Time Scales: An Introduction with Applications. Birkhäuser, Basel (2001)

4. Bohner, M., Georgiev, S.G.: Multivariable Dynamic Calculus on Time Scales. Springer, Cham (2016)

5. Bohner, M., Peterson, A.: Advances in Dynamic Equations on Time Scales. Birkhäuser, Basel (2003)

6. Hopf, E.: Uber die Zusammenhänge zwischen gewissen höheren Differe nzenquotienten reeller Funktionen einer reellen Variablen und deren Differenzierbarkeitseigenschaften. Dissertation, Univ. Berlin (1926)

7. Popoviciu, T:: Sur quelques propriétés des fonctions d'une ou de deux variables réelles. Thése, Faculté des Sciences de Paris, (1933). See also Mathematica (Cluj) 8, 1-85 (1934)

8. Popoviciu, T:: Les Fonctions Convexes. Hermann, Paris (1944)

9. Pečarić, J., Proschan, F., Tong, Y.L.: Convex Functions, Partial Orderings, and Statistical Applications. Academic Press, San Diego (1992) 
10. Bullen, P.: A criterion for n-convexity. Pac. J. Appl. Math. 36(1), 81-98 (1971)

11. Dinu, C.: Convex functions on time scales. An. Univ. Craiova, Ser. Mat. Inform. 35, 87-96 (2008)

12. Agarwal, R.P., O'Regan, D., Saker, S.H.: Hardy Type Inequalities on Time Scales. Springer, Berlin (2016)

13. Agarwal, R.P., O'Regan, D., Saker, S.H.: Dynamic Inequalities on Time Scales, vol. 2014. Springer, Cham (2014)

14. Rozarija, M., Pečarić, J.: Jensen-type inequalities on time scales for $n$-convex functions. Commun. Math. Anal. 21(2), 46-67 (2018)

15. Agarwal, R.P.: Difference Equations and Inequalities-Theory, Methods, and Applications. CRC Press, Boca Raton (2000)

16. Varberg, D.E., Roberts, A.W.: Convex Functions. Academic Press, New York (1973)

17. Bullen, P.: Dictionary of Inequalities, p. 191. Chapman \& Hall/CRC, London (2015)

18. Isaacson, E., Keller, H.B.: Analysis of Numerical Methods. Courier Corporation (2012)

\section{Submit your manuscript to a SpringerOpen ${ }^{\circ}$} journal and benefit from:

- Convenient online submission

- Rigorous peer review

- Open access: articles freely available online

- High visibility within the field

- Retaining the copyright to your article

Submit your next manuscript at $\gg$ springeropen.com 